\title{
Multiscale Finite Element Method Based on Local Preconditioning for Compressible Flows at Low Mach Numbers
}

\author{
Sérgio Souza Bento ${ }^{1}$ \\ Departamento de Matemática Aplicada - UFES, São Mateus \\ Leonardo Muniz de Lima ${ }^{2}$ \\ Instituto Federal do Espírito Santo - IFES, Aracruz \\ Isaac Pinheiro Santos ${ }^{3}$ \\ Departamento de Matemática Aplicada - UFES, São Mateus \\ Lucia Catabriga ${ }^{4}$ \\ Departamento de Informática - UFES, Vitória
}

\begin{abstract}
In this work we present a nonlinear multiscale finite element method coupled with the Weiss-Smith/Choi-Merkle (WSCM) local preconditioner for solving steady compressible flows at low Mach numbers. The multiscale formulation is based on the strategy of separating scales, in which the subgrid scale space is spanned by bubble functions, allowing to use a static condensation procedure in the local matrix system to define the resolved scale problem. The resulting numerical formulation is completed by adding an artificial viscosity operator in all scales of the discretization. We evaluate the multiscale formulation coupled with the WSCM preconditioner comparing it with the non-preconditioned case. The numerical experiments show that this numerical methodology yields good results.
\end{abstract}

Keywords. Multiscale formulation. Local preconditioning. Compressible flows. Euler equations.

\section{Introduction}

It is well known that the numerical solution of compressible flow problems at low Mach numbers, modelled by Euler equations, is a very challenging problem. As mentioned in [1], most of the numerical methods for solving compressible flows at low-Mach numbers are based on the local preconditioning strategy of the Euler equations. Local preconditioning or preconditioning mass matrix scheme consists of premultiplying the time derivative by an appropriate matrix in order to uniform the eigenvalues, smoothing the discrepancy of the different time scales $[2,3]$. It is applied to the set of continuous equations before any

\footnotetext{
${ }^{1}$ sergio.bento@ufes.br.

${ }^{2}$ lmuniz@ifes.edu.br.

3 isaac.santos@ufes.br.

${ }^{4}$ luciac@inf.ufes.br.
} 
discretization is done. However, the original problem and the preconditioned one have different time evolution but the same steady-state solution.

Bento et al. [4] proposed a density-based multiscale finite element method, called NMV (Nonlinear Multiscale Viscosity) method, for solving compressible flows, yielding good results for flows in transonic and supersonic regimes. Numerical methods for solving low speed flow are usually pressure-based, since that density-based strategy suffers severe deficiencies when applied to this kind of problem, degrading convergence speeds, and impacting the efficiency and accuracy of the numerical formulations.

In this work we combine the nonlinear density-based NMV method with the WeissSmith/Choi-Merkle (WSCM) local preconditioner, presented in [2], for solving compressible flows at low Mach numbers. The numerical experiments show that this numerical methodology yields good results.

\section{Governing equations and multiscale formulation}

The two-dimensional compressible Euler equations may be written with conservative variables in the quasi-linear form,

$$
\frac{\partial \mathbf{U}}{\partial t}+\mathbf{A}_{\mathbf{x}} \frac{\partial \mathbf{U}}{\partial x}+\mathbf{A}_{\mathbf{y}} \frac{\partial \mathbf{U}}{\partial y}=\mathbf{0}, \text { in } \Omega \times\left(0, t_{f}\right]
$$

where $t_{f}$ is a positive real number, representing the final time and $\Omega$ is a bounded domain in $\mathbb{R}^{2}$, with boundary $\Gamma, \mathbf{U} \in \mathbb{R}^{4}$ is the vector of conservative variables defined by

$$
\mathbf{U}=\underbrace{\left[\begin{array}{c}
U_{1} \\
U_{2} \\
U_{3} \\
U_{4}
\end{array}\right]}_{\text {conservative variables }}=\underbrace{\left[\begin{array}{c}
1 \\
u \\
v \\
E
\end{array}\right]}_{\text {primitive variables }},
$$

where $\rho$ is the fluid density, $\mathbf{u}=\left[\begin{array}{ll}u & v\end{array}\right]^{T}$ is the velocity vector, $\rho E$ is the total energy, and $E$ is the total specific energy. $\mathbf{A}_{x}=\frac{\partial \mathbf{F}_{x}}{\partial \mathbf{U}}$ and $\mathbf{A}_{y}=\frac{\partial \mathbf{F}_{y}}{\partial \mathbf{U}}$ are the Jacobian matrices and $\mathbf{F}$ is the Euler flux vector. Others important physical quantities are the pressure $p$ and the Mach number, $M=\frac{\|\mathbf{u}\|_{2}}{c}$, where $c=\sqrt{\gamma \frac{p}{\rho}}$ is the speed of sound, with $\gamma=\frac{c_{p}}{c_{v}}(\gamma>1)$ being the ratio of specific heats, and $c_{p}$ and $c_{v}$ are the coefficients of specific heat at constant pressure and volume, respectively. Associated with Eq.(1) we have an appropriate set of boundary and initial conditions.

To define the multiscale finite element method, we consider a triangular partition $\mathcal{T}_{h}$ of the domain $\Omega$ into nel elements, where $\Omega=\bigcup_{e=1}^{n e l} \Omega_{e}$ with $\Omega_{i} \cap \Omega_{j}=\emptyset$, for $i, j=$ $1,2, \ldots$, nel and $i \neq j$. We introduce the function space $\mathcal{V}_{Z h b}$, which is written as the direct sum, $\mathcal{V}_{Z h b}=\mathcal{V}_{Z h} \oplus \mathcal{V}_{b}$ where the subspaces $\mathcal{V}_{Z h}$ and $\mathcal{V}_{b}$ are given by

$$
\begin{aligned}
\mathcal{V}_{Z h}=\left\{\mathbf{U}_{h} \in\left[H^{1}(\Omega)\right]^{4} ;\right. & \left.\left.\mathbf{U}_{h}\right|_{\Omega_{e}} \in\left[\mathbb{P}_{1}\left(\Omega_{e}\right)\right]^{4}, \mathbf{B} \mathbf{U}_{h}=\mathbf{Z} \text { on } \Gamma_{D}\right\}, \\
\mathcal{V}_{b} & =\left\{\mathbf{U}_{b} \in\left[H_{0}^{1}(\Omega)\right]^{4} ;\left.\quad \mathbf{U}_{b}\right|_{\Omega_{e}} \in\left[\operatorname{span}\left(\psi_{b}\right)\right]^{4}, \quad \forall \Omega_{e} \in \mathcal{T}_{h}\right\}
\end{aligned}
$$


with $\mathbb{P}_{1}\left(\Omega_{e}\right)$ representing the set of first order polynomials in $\Omega_{e}, H^{1}(\Omega)$ denotes the Sobolev space of square-integrable functions whose first derivatives are also square-integrable, $H_{0}^{1}(\Omega)$ is a space of function in $H^{1}(\Omega)$ that vanish at the boundary of $\Omega$, and $\psi_{b}$ is a bubble function. The space $\mathcal{V}_{Z h}$ represents the resolved (coarse) scale space whereas $\mathcal{V}_{b}$ stands for the subgrid (fine) scale space. $\mathbf{B}$ denotes a general boundary operator, and $\mathbf{Z}$ is a given function.

The nonlinear multiscale method proposed in [4], called Nonlinear Multiscale Viscosity (NMV) method, adds artificial viscosity isotropically in all scales of the discretization, where the amount of artificial viscosity is given by the $\mathrm{YZ} \beta$ shock-capturing viscosity parameter, presented in [5]. The NMV method for the Euler equation consists of finding $\mathbf{U}_{h b}=\mathbf{U}_{h}+\mathbf{U}_{b} \in \mathcal{V}_{Z h b}$ with $\mathbf{U}_{h} \in \mathcal{V}_{Z h}, \mathbf{U}_{b} \in \mathcal{V}_{b}$ such that

$$
\begin{gathered}
\underbrace{\int_{\Omega} \mathbf{W}_{h b} \cdot\left(\frac{\partial \mathbf{U}_{h b}}{\partial t}+\mathbf{A}_{x}^{h} \frac{\partial \mathbf{U}_{h b}}{\partial x}+\mathbf{A}_{y}^{h} \frac{\partial \mathbf{U}_{h b}}{\partial y}\right) d \Omega}_{\text {Galerkin term }}+ \\
\underbrace{\sum_{e=1}^{n e l} \int_{\Omega_{e}} \delta_{h}\left(\mathbf{U}_{h}\right)\left(\frac{\partial \mathbf{W}_{h b}}{\partial x} \cdot \frac{\partial \mathbf{U}_{h b}}{\partial x}+\frac{\partial \mathbf{W}_{h b}}{\partial y} \cdot \frac{\partial \mathbf{U}_{h b}}{\partial y}\right) d \Omega}_{\text {Nonlinear stabilization term in all scales }}=\mathbf{0}, \quad \forall \mathbf{W}_{h b} \in \mathcal{V}_{0 h b},
\end{gathered}
$$

where $\mathbf{W}_{h b}=\mathbf{W}_{h}+\mathbf{W}_{b} \in \mathcal{V}_{0 h b}$ with $\mathbf{W}_{h} \in \mathcal{V}_{0 h}, \mathbf{W}_{b} \in \mathcal{V}_{b}$ and the amount of artificial viscosity, $\delta_{h}\left(\mathbf{U}_{h}\right)$, is calculated on the element-level by using the YZ $\beta$ shock-capturing viscosity parameter [5],

$$
\delta_{h}\left(\mathbf{U}_{h}\right)=\left\|\mathbf{Y}^{-1} R\left(\mathbf{U}_{h}\right)\right\|\left(\sum_{i=1}^{2}\left\|\mathbf{Y}^{-1} \frac{\partial \mathbf{U}_{h}}{\partial x_{i}}\right\|_{2}^{2}\right)^{\frac{\beta}{2}-1}\left\|\mathbf{Y}^{-1} \mathbf{U}_{h}\right\|^{1-\beta} h^{\beta},
$$

where

$$
R\left(\mathbf{U}_{h}\right)=\frac{\partial \mathbf{U}_{h}}{\partial t}+\mathbf{A}_{x}^{h} \frac{\partial \mathbf{U}_{h}}{\partial x}+\mathbf{A}_{y}^{h} \frac{\partial \mathbf{U}_{h}}{\partial y}
$$

is the residue of the problem on $\Omega_{e}, \mathbf{Y}$ is a diagonal matrix constructed from the reference values of the components of $\mathbf{U}, h$ is the local length scale defined as follow $h=$ $\left(\sum_{a=1}^{3}\left|\mathbf{j} \cdot \nabla N_{a}\right|\right)^{-1}, \mathbf{j}$ is a unit vector defined as $\mathbf{j}=\nabla \rho /\|\nabla \rho\|_{2}$ and $N_{a}$ is the interpolation function associated with node $a$. The local length $h$ is defined automatically taking into account the directions of high gradients and spatial discretization domain.

\section{Local preconditioning for the Euler equations}

As mentioned in the introduction section, the local preconditioning is applied to the set of continuous equations before any discretization is done. Denoting by $\mathbf{P}$ the (nonsingular) preconditioning matrix, then the system of equations (1) after the preconditioning process reads 


$$
\mathbf{P}^{-1} \frac{\partial \mathbf{U}}{\partial t}+\mathbf{A}_{\mathbf{x}} \frac{\partial \mathbf{U}}{\partial x}+\mathbf{A}_{\mathbf{y}} \frac{\partial \mathbf{U}}{\partial y}=\mathbf{0} \quad \text { or } \quad \frac{\partial \mathbf{U}}{\partial t}+\mathbf{P} \mathbf{A}_{\mathbf{x}} \frac{\partial \mathbf{U}}{\partial x}+\mathbf{P} \mathbf{A}_{\mathbf{y}} \frac{\partial \mathbf{U}}{\partial y}=\mathbf{0}, \text { in } \Omega \times\left(0, t_{f}\right]
$$

The solutions of problems (1) and (8) evolve in time differently, but converge in time to the same steady-state solution, since the time derivatives go to zero.

In this work we study the Weiss-Smith/Choi-Merkle (WSCM) local preconditioner, presented in [2]. This preconditioner is based on the symmetric Weiss-Smith (WS) [6] and on the viscous $\mathrm{CM}[7,8]$ preconditioners. An explicit expression for the WSCM preconditioner in conservative variables $[2]$ is

$$
\mathbf{P}=\mathbf{I}+\alpha\left[\begin{array}{cccc}
\theta & -u & -v & 1 \\
u \theta & -u u & -u v & u \\
v \theta & -u v & -v v & v \\
H \theta & -u H & -v H & H
\end{array}\right]
$$

where

$$
\alpha=\frac{\gamma-1}{c^{2}}[(1-\delta) \epsilon-1], \quad \theta=\frac{1}{2}\|\boldsymbol{u}\|^{2}+\delta \frac{\epsilon c^{2}}{(\gamma-1)[(1-\delta) \epsilon-1]}
$$

and $H$ is the total enthalpy. The parameter $\delta \in[0,1]$; for $\delta=0$, the preconditioner is the WS preconditioner, whereas for $\delta=1$ the CM is recovered. For Euler equations, the preconditioning parameter $\epsilon$ is given by

$$
\epsilon=\min \left\{1, \max \left\{M_{l i m}^{2}, M^{2}, \sigma_{p g r} \frac{|\Delta p|}{\rho c^{2}}\right\}\right\}
$$

where $M_{\text {lim }}^{2}=10^{-5}, \sigma_{p g r}=2$, and $\delta=0.5$ according to [2]. We define the maximum pressure variation $(\Delta p)$ on the triangle as

$$
|\Delta p|=\max \left\{\left|p_{1}-p_{2}\right|,\left|p_{1}-p_{3}\right|,\left|p_{2}-p_{3}\right|\right\}
$$

where $p_{i}$ is the pressure on the node $i=1,2,3$.

\section{Numerical results}

The flow over an airfoil is an attractive problem to analyze the numerical instability arising from low Mach number values, that occurs in the Euler equations. This section shows the results of a flow passing through a NACA 0012 airfoil at an angle of attack of $0^{\circ}$ and varying inflow Mach number $M=0.01,0.1$ and 0.3 .

The experiment was executed taking into a account an unstructured triangular mesh of 5,606 elements and 2,886 nodes, in the computational domain given by a circle centered at the $(0,0)$ with radius 15 (Fig. 1). Aiming to avoid numerical instabilities of reflecting waves [3], we consider a distance ahead the leading edge of the airfoil to the inflow and outflow boundaries. The inflow data is set up as $\rho=1.0, \mathbf{u}=\left[\begin{array}{ll}1.0 & 0.0\end{array}\right]^{T}$, and $\mathrm{T}=1.0$, where $T$ is the temperature. 


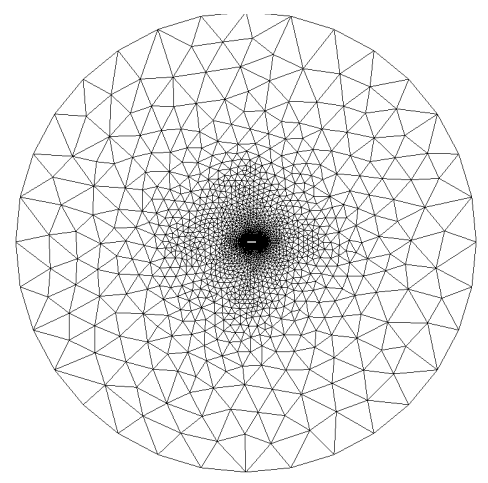

(a) NACA 0012 mesh

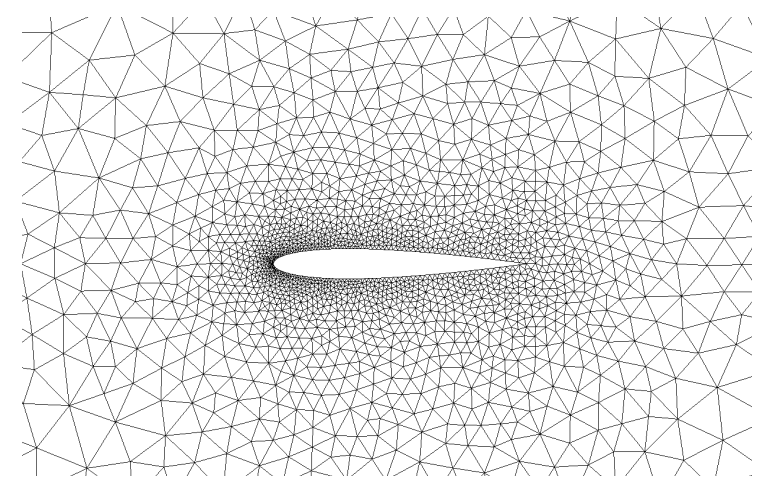

(b) Detail of the NACA 0012 airfoil

Figure 1: Unstructured triangular mesh of 5,606 elements and 2,886 nodes.

The numerical solution is advanced in time by the predictor-corrector algorithm adapted for the multiscale framework in [4] for the Euler equations. A restarted version of the GMRES solver is used to find the solution of the linearized system in each nonlinear and time iterations. The GMRES parameters are: 30 vectors to the restart process and tolerance equals $10^{-5}$. The time-step size is $10^{-3}$ and the simulation runs until $t_{f}=20.0(20,000$ steps), and 3 fixed nonlinear iterations. In this example, we evaluate the WSCM preconditioner comparing it with the non-preconditioned (NP) case. The tests are carried out with the intention of analyzing accuracy issues, specially in the incompressibility limit. Due to flow at a low speed to demonstrate an incompressible behavior, i.e., density variation is almost negligible, we use the pressure contour to analyze this experiments.

Figures 2, 3 and 4 show the pressure contours for inflow Mach number $M=0.01,0.1$ and 0.3 , respectively. As happened in the work of [3] for the non-preconditioned case, our multiscale methodology does not work in the low Mach number limit, i.e., when the Mach number approaches to zero. In the low Mach number flows the numerical methods based on conservative variables suffer with undesirable effects [9], because the flow demonstrates an incompressible behavior. The numerical solutions without local preconditioning in the low Mach number limit are completely oscillatory, e.g. Fig. 2(a). On the other hand, the NMV method coupled with local preconditioned is able to solve problems with an incompressible behavior, as shown in Fig. 2(b), 3(b), and 4(b).

\section{Conclusions}

We applied the NMV method coupled with the Weiss-Smith/Choi-Merkle (WSCM) local preconditioner for solving the NACA 0012 airfoil problem at low Mach numbers. We simulated the flow over the NACA 0012 airfoil under the following regimes of inflow Mach numbers: $0.01 ; 0.1 ; 0.3$. The solutions obtained with the NMV without local preconditioning are completely oscillatory in the low Mach number limit (e.g. for $M=0.01$ ), since methods based on conservative variables fail in this case. On the other hand, our numerical methodology combined with local preconditioning exhibited promising results 


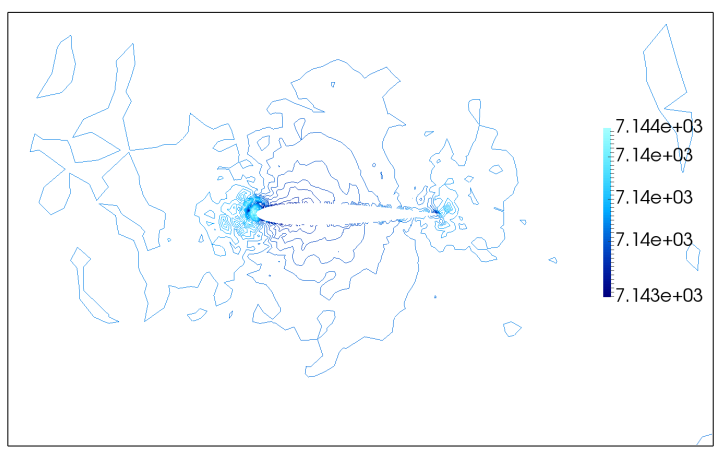

(a) $\operatorname{NMV}(\mathrm{NP})$

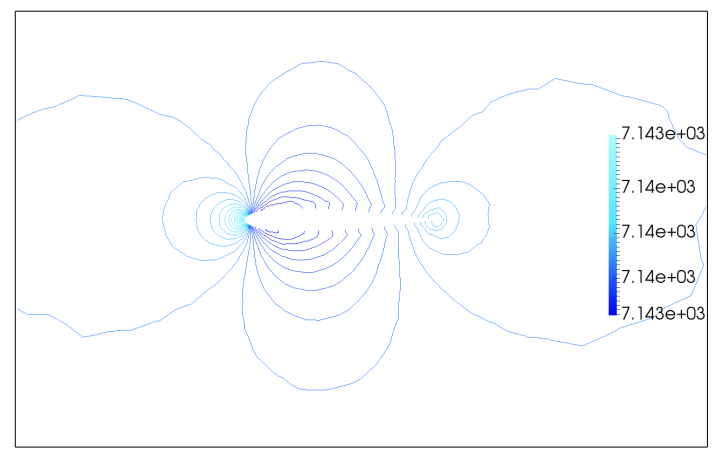

(b) NMV(WSCM)

Figure 2: NACA 0012: Pressure contours for $M=0.01$ at the inflow.

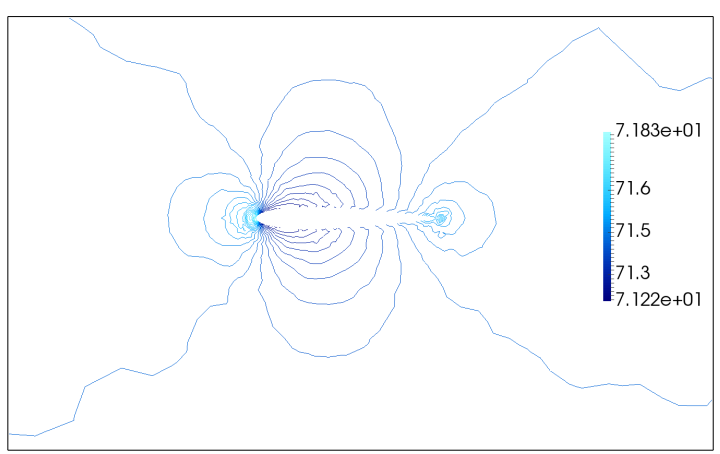

(a) $\operatorname{NMV}(\mathrm{NP})$

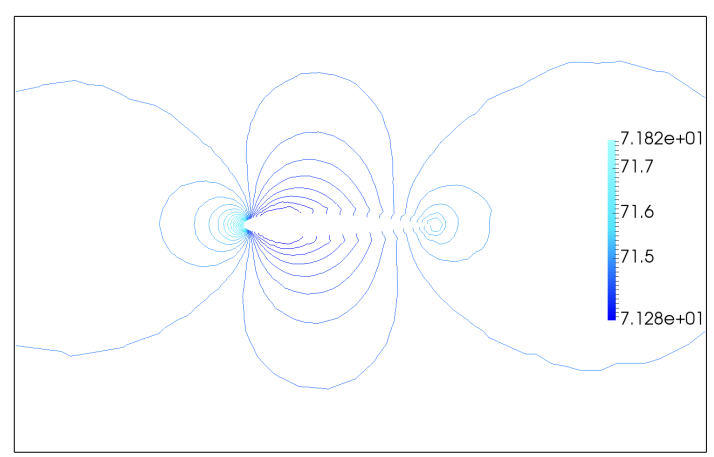

(b) NMV(WSCM)

Figure 3: NACA 0012: Pressure contours for $M=0.1$ at the inflow.

to this problem.

\section{Acknowledgments}

The authors would like to thank the support through the Espírito Santo State Research Support Foundations (FAPES).

\section{References}

[1] M. Salinas-Vázquez, W. Vicente, E. Barrios, E. Martínez, A. Palacio, and A. Rodríguez. A low-Mach number method for the numerical simulation of complex flows. Applied Mathematical Modelling, 37(22):9132 - 9146, 2013.

[2] Y. Colin, H. Deniau, and J.-F. Boussuge. A robust low speed preconditioning formulation for viscous flow computations. Computers \& Fluids, 47(1):1-15, 2011. 


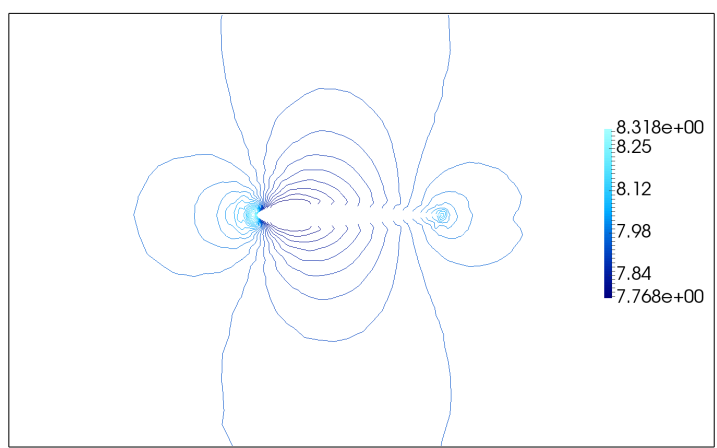

(a) $\mathrm{NMV}(\mathrm{NP})$

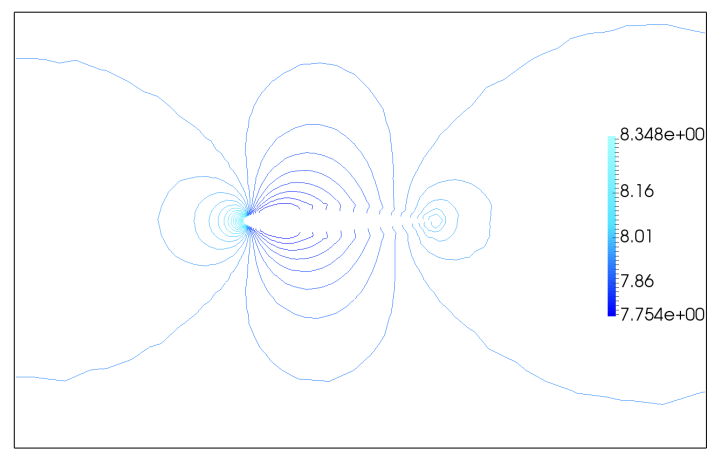

(b) NMV(WSCM)

Figure 4: NACA 0012: Pressure contours for $M=0.3$ at the inflow.

[3] M. M. Ginard, M. Vázquez, and G. Houzeaux. Local preconditioning and variational multiscale stabilization for Euler compressible steady flow. Computer Methods in Applied Mechanics and Engineering, 305:468 - 500, 2016.

[4] S. S. Bento, L. M. de Lima, R. Z. Sedano, L. Catabriga, and I. P. Santos. A nonlinear multiscale viscosity method to solve compressible flow problems. In Computational Science and Its Applications - ICCSA 2016 - 16th International Conference, Beijing, China, July 4-7, 2016, Proceedings, Part I, pages 3-17, 2016.

[5] T. E. Tezduyar and M. Senga. Stabilization and shock-capturing parameters in SUPG formulation of compressible flows. Computer Methods in Applied Mechanics and Engineering, 195(13-16):1621-1632, 2006.

[6] J. M. Weiss and W. A. Smith. Preconditioning applied to variable and constant density flows. AIAA Journal, 33(11):2050 - 2057, 1995.

[7] Y.-H. Choi and C. L. Merkle. The application of preconditioning in viscous flows. Journal of Computational Physics, 105(2):207 - 223, 1993.

[8] M. M. Ginard, G. Bernardino, M. Vázquez, and G. Houzeaux. Fourier stability analysis and local Courant number of the preconditioned variational multiscale stabilization (P-VMS) for Euler compressible flow. Computer Methods in Applied Mechanics and Engineering, 301:28 - 51, 2016.

[9] Z. Li and H. Xiang. Development of a Navier-Stokes flow solver for all speeds on unstructured grids. Engineering Letters, 21(2):89-94, 2013. 